•综述・

\title{
食物网结构与功能：理论进展与展望
}

\author{
王少鹏*
}

(北京大学城市与环境学院生态研究中心, 地表过程分析与模拟教育部重点实验室, 北京 100871)

\begin{abstract}
摘要: 食物网刻画了物种间通过捕食而形成的复杂网络关系。阐明食物网结构与功能之间的关系, 既是生态学的 基本理论问题, 也是预测全球变化背景下生态系统响应的重要依据。早期关于食物网结构与功能的研究往往是分 离的, 或是基于食物链等的简单网络模型, 而近期研究基于复杂食物网模型取得了重要理论进展。本文综述了食 物网研究的理论方法和近期进展, 特别介绍了复杂食物网中的结构、多样性和功能的度量指标、结构-多样性-功 能之间的关系以及全球变化对食物网结构与功能的影响。本文最后对未来的一些研究方向进行了展望, 包括与功 能性状和化学计量学的整合、食物网与其他网络类型的整合以及拓展食物网研究的空间和时间尺度。
\end{abstract}

关键词: 杂食性; 模块性; 水平多样性; 垂直多样性; 能量流

\section{Food web structure and functioning: Theoretical advances and outlook}

\author{
Shaopeng Wang* \\ Institute of Ecology, College of Urban and Environmental Sciences, Key Laboratory for Earth Surface Processes of the \\ Ministry of Education, Peking University, Beijing 100871
}

\begin{abstract}
The relationship between food web structure and functioning can have important implications for predicting the responses of ecosystems to global changes. Previous studies have mainly explored food web structure and functioning separately or in simple food web models (e.g. food chains), but recent studies made significant progress in understanding the structure and functioning of complex food webs. This paper reviews the theoretical approaches and recent advances of complex food webs. In particular, I summarize the multiple metrics for quantifying the structure, biodiversity, and functioning of complex food webs, explain the theoretical framework for modeling complex food webs, and review the recent progress in understanding the relationships between food web structure, biodiversity, and functioning, and how they respond to global changes. I end with discussion on potential future directions by integrating food web theory with functional trait, ecological stoichiometry, other types of ecological networks, metacommunity theory, and evolutionary models.
\end{abstract}

Key words: omnivory; modularity; horizontal diversity; vertical diversity; energy flux

生态网络刻画了生态系统中生物组分之间的 相互作用。食物网是生态学中研究最早、最多的网 络类型, 它表征了物种之间通过捕食而形成的复杂 网络关系。食物网表明生态系统的营养结构和过程 具有高度的复杂性, 这一复杂性是生态系统进行物 质循环和能量流动的基础 (Lindeman, 1942; Bascompte, 2009; Thompson et al, 2012)。阐明食物 网结构与功能之间的关系, 既是生态学的基本理论 问题, 也是预测全球变化背景下生态系统响应的重
要依据(Tylianakis et al, 2008; Sentis et al, 2014)。

虽然食物网是生态学研究的一个经典议题, 但 早期关于食物网结构和功能的研究往往是分离的。 自Elton (1927)发表Animal Ecology一书以来, 群落 生态学家关于食物网的拓扑结构、不同营养级的数 量特征等开展了大量研究。随后, Lindeman (1942) 关于湖泊生态系统营养动态的研究发表, 引发了生 态系统生态学家对食物网能量流动、营养传递效率 和生产力等的广泛研究。过去几十年来, 生态学家

收稿日期: 2020-06-15; 接受日期: 2020-07-26

基金项目: 国家自然科学基金(31988102; 31870505)和科技部重点研发计划(2017YFC0503906)

* 通讯作者 Author for correspondence. E-mail: shaopeng.wang@pku.edu.cn 
尝试将食物网结构与功能结合起来, 但这些研究大 多关注简单网络模块, 比如两个或三个物种构成的 食物链或竞争模块 (Pimm，1982; Loreau，2010; McCann, 2012)。这些简单模块只是整个食物网的一 个子集，基于此类子集的研究结果并不一定能推广 至复杂食物网(Wang \& Brose, 2018)。为了理解自然 生态系统整体的结构和功能, 需要开展复杂食物网 研究(Seibold et al, 2018)。然而, 食物网的高度复杂 性为研究工作带来了很大的挑战, 尤其是经验分 析。因此, 为了理解复杂食物网的结构和功能及其 应对外界干扰的稳定性, 生态学家常常借助理论模 型方法进行研究(May, 1973; McCann, 2012; Moore \& de Ruiter, 2012)。

本文对近期关于复杂食物网结构与功能的理 论进展做了综述。首先介绍复杂食物网中网络结 构、多样性和功能的度量指标, 接着围绕以下三个 问题对近期进展做了概述(图1)：食物网结构如何 影响物种多样性维持? 食物网结构与多样性如何 影响生态系统功能? 全球变化如何改变食物网结 构、多样性与功能? 本文所介绍的理论研究主要基 于复杂食物网模型, 在Box 1中对此类模型的基本 框架做了阐述。需要说明的是, 复杂性与稳定性关 系是食物网研究中的一个经典和重要问题, 关于这
一问题已有多篇中文综述(陈云峰等, 2014; 徐光华 等, 2019), 因此本文不作特别介绍。

\section{食物网结构、多样性和功能的度}

\section{1 食物网结构指标}

生态学家提出了大量指标来刻画食物网的结 构(Gauzens et al, 2013; 孙书存, 2017)。在整个食物 网水平上，三个量化生态系统复杂性的常用指标是： 总物种数 $(\mathrm{S}$, 即食物网中所有动物和植物的物种 数)、总连接数( $\mathrm{L}$, 即食物网中具有捕食关系的所有 连接数)和连通度(connectance, $\left.\mathrm{L} / \mathrm{S}^{2}\right)$ 。此外, 模块性 (modularity) 度量了食物网的分室特征 (compartment), 具体指食物网内由不同物种集合形 成的子模块，模块内物种有较紧密的交互关系，模 块间的交互关系相对较少或较弱(Yodzis，1982; Krause et al, 2003)。

在物种水平上, 可以定义物种的连接度(degree, 即与该物种具有捕食关系的所有捕食者和猎物的 物种数)、广食性(generality, 即该物种的所有猎物种 数)、脆弱性(vulnerability, 即该物种的所有捕食者 种数)等。对于食物网中的物种来说，一个核心概念 是物种的营养级(trophic level)。营养级刻画了物种 在生态系统中的营养位置, 通常定义为从非生物资

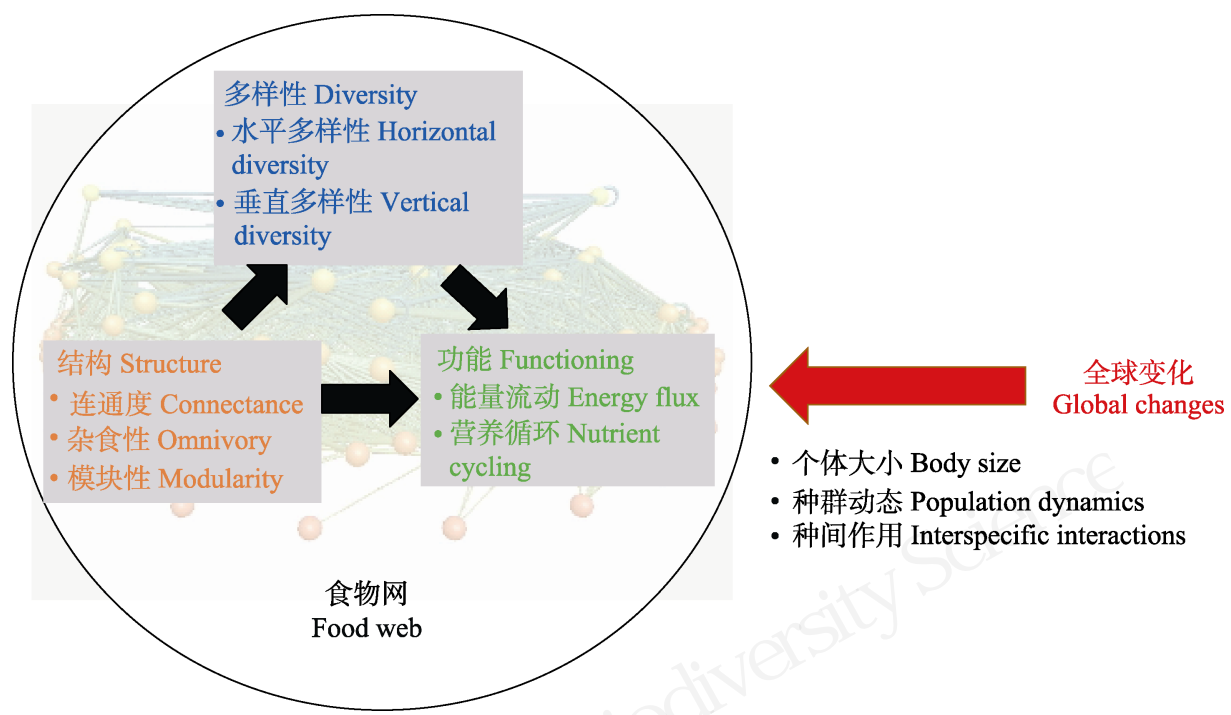

图1 复杂食物网的结构、多样性与功能间的关系。食物网结构可影响物种多样性和生态系统功能的维持, 多样性也对功能 起重要作用。全球变化可通过改变个体大小、种群动态、种间作用等影响食物网结构、多样性与功能。背景食物网来自 http://www.foodwebs.org/。

Fig. 1 Relationships between the structure, diversity and functioning of complex food webs. Food web structure can affect species diversity and ecosystem functioning, and diversity also affects functioning. Global change can affect food webs through changing species body size, population dynamics, interspecific interaction, etc. The food web in the background was obtained from http://www.foodwebs.org/. 


\section{Box 1 复杂食物网理论模型}

食物网建模的核心是对网络结构与种群动态的理论刻画。网络结构决定了每个物种在生态系统中的位置, 即“谁吃谁” (Who eats who?)。种群动态刻画了任一物种如何受其捕食者和猎物的影响，即“如何吃” (How does a predator eat its prey?)。 这里概述食物网结构与动态模型的一般框架。在具体应用中, 针对研究问题可有不同的具体模型。比如在复杂性一稳定性 研究中，陈云峰等(2014)和徐光华等(2019)综述了多种常用的食物网模型。

\section{- “谁吃谁”: 模拟食物网结构}

生态学家提出了多种理论模型试图刻画食物网的网络结构。早期的理论模型包括随机模型(May, 1973)和级联模型 (cascade model; Cohen \& Newman, 1985)。随机模型假定生态系统内不同物种之间的捕食关系是随机发生的, 往往只作为一 个零模型来使用。比如, May (1973)关于生态系统稳定性与复杂性关系的研究就使用了随机模型。级联模型考虑了物种间 的非对称性，假定物种可在某一性状轴上排序，性状值高的物种可捕食性状值低的物种，反之则不成立。该模型假定的性 状轴可以是影响捕食关系的特定性状, 比如个体大小。然而有研究表明, 个体较大的物种并非取食所有比其小的物种, 而 是有一个最优取食范围(Persson et al, 1998)。基于这一考虑, Williams和Martinez (2000)提出了生态位模型(niche model)。该 模型在级联模型的基础上，假定捕食者只捕食某一性状范围内的物种，且允许自食和互相捕食。生态位模型为自然界食物 网的多种结构指标给出了很好的解释, 因此成为食物网研究的一个经典模型。后续研究基于生态位模型发展了很多变种, 从不同角度对生态位模型做了扩展，包括引入谱系关系(Cattin et al, 2004)、多维生态位(Allesina et al, 2008)、个体大小限制 (Schneider et al, 2016)等。需要注意的是，物种分布模型中同样有“生态位模型”，用以刻画物种对环境因子的响应方式，与 此处模拟食物网结构的生态位模型不同。

\section{- “如何吃”: 模拟食物网动态与功能}

对食物网中的任一物种而言, 种群增长主要取决于其获取资源的速率, 种群下降主要取决于其被捕食的速率以及新 陈代谢和自然死亡。因此, 种群动态模拟的关键是给出物种获取资源或捕食的速率。通常地, 这一速率由功能响应 (functional response)函数刻画(Holling, 1959):

$$
F(N)=\frac{a N^{q}}{1+a h N^{q}}
$$

其中, $N$ 表示猎物密度, $F(N)$ 表示单个捕食者的捕食速率, $a$ 表示捕食者的攻击速率(attack rate), $h$ 表示消化或处理一只猎物所 需的时间 (handling time)。 $q$ 是一个调控密度依赖形式的参数。当 $q=0$ 时，捕食速率 $F(N)$ 是与猎物密度 $N$ 无关的常数，称为 “I 型”功能响应; $q=1$ 时, $F(N)$ 在密度 $N$ 较低时随 $N$ 线性增长, 随后增长速率减慢, 最终达到饱和水平, 称为“II型”功能响应; $q$ $=2$ 时, $F(N)$ 在密度 $N$ 较低时随 $N$ 呈二次函数增长, 随后增长减慢, 最终达到饱和水平, 称为“III型”功能响应。很多研究者通 过实验分析了猎物密度和捕食速率之间的关系，发现很多实验系统都服从“II型”功能响应(Rall et al, 2012)。但近期也有实 验表明，功能响应的类型受物种属性的影响：当捕食者与猎物个体大小接近时，其捕食过程服从“II型”功能响应; 而当捕 食者比猎物大很多时, 服从“III型”功能响应(Kalinkat et al, 2013)。虽然以上三种功能响应函数最为常用, 但生态学家也提出 了很多其他模型来刻画捕食速率和猎物密度的关系(Turchin, 2003; Yan \& Zhang, 2014)。

基于功能响应函数, 食物网中任一植物种 $i\left(P_{i}\right)$ 和动物种 $j\left(A_{j}\right)$ 的种群大小的时间动态由以下方程决定(Schneider et al, 2016):

$$
\begin{aligned}
& \frac{d P_{i}}{d t}=r_{i} G_{i} P_{i}-\sum_{k} A_{k} F_{k i}-x_{i} P_{i} \\
& \frac{d A_{j}}{d t}=e_{1} A_{j} \cdot \sum_{i: \text { 植物资源动物资源 }} F_{j}+e_{j} A_{j} \cdot \sum F_{j k}-\sum_{l} A_{i} F_{l j}-x_{j} A_{j}
\end{aligned}
$$

其中, $r_{i}$ 表示植物种 $i$ 的最大生长速率, $G_{i}$ 刻画了营养元素对植物种 $i$ 生长的限制(见Schneider et al, 2016), $x_{i}$ 表示个体代谢速 率; $e_{1}$ 和 $e_{2}$ 分别是取食植物和动物时的能量利用效率。 $F_{j i}$ 表示功能响应函数, 刻画了捕食者 $j$ 对其猎物种 $i$ 的取食速率。但不 同于公式B1，由于捕食者 $j$ 有多种猎物，因此功能响应函数扩展为下式(Schneider et al, 2016):

$$
F_{j i}=\frac{\omega_{j i} a_{j i} N_{i}^{q}}{1+\sum_{k} \omega_{j k} a_{j k} h_{j k} N_{k}^{q}}
$$

其中 $N_{i}$ 表示第 $i$ 个猎物种的生物量, $a_{j k}$ 和 $h_{j k}$ 表示捕食者 $j$ 对猎物 $k$ 的攻击速率和处理时间, $\omega_{j k}$ 表示捕食者 $j$ 对猎物 $k$ 的取食偏好。 类似地, $q$ 表示取食速率对猎物密度的依赖关系为 II型 $(q=1)$ 或III型 $(q=2)$ 功能响应。

为了模拟食物网功能, 后续研究通过引入物种个体大小, 将基于种群的模型拓展到基于生物量的模型, 其中关注变 量不再是“种群变化”，而是“生物量变化” (Yodzis \& Innes, 1992; Brose et al, 2006)。这一拓展将捕食过程直接描述为能量流 动过程, 从而构建了食物网种群动态与生态系统功能之间的联系。此后基于大量食物网的数据分析表明, 个体大小对食物 网结构(捕食关系)和动态(代谢速率、捕食过程)均有重要影响(Brose et al, 2006; Rall et al, 2012)。在此基础上, Schneider等 (2016)发展了一个新的基于个体大小的参数化食物网模型, 其中食物网结构和动态均由个体大小决定。该模型整合了物种 性状、代谢理论、能量动态等, 为研究食物网结构和功能提供了一个新的理论工具(Wang \& Brose, 2018)。 
源到该物种的能量传递路径的长度。如果该物种取 食多种资源，那么可根据其与非生物资源的不同传 递路径定义其营养级: 最短链营养级、最长链营养 级、平均营养级(Williams \& Martinez, 2004)。当所 有路径长度都相同时，三种营养级的定义是一致的; 反之, 则意味着不同传递路径长度不同, 三种营养 级的计算结果也不同。产生这种差异的原因即物种 的杂食性(omnivory), 它指的是食物网中的某些物 种可以取食位于两个(或多个)不同营养级的物种 (Pimm, 1982)。狭义上的杂食性指某种动物可取食 植物和动物, 而食物网研究中一般采用广义的杂食 性定义, 即可以取食位于不同营养级的两物种。研 究发现, 自然界食物网中存在广泛的杂食性 (Thompson et al, 2007)。与杂食性相对应, 有学者提 出了营养一致性(trophic coherence)指标(Johnson et al, 2014)。该指标度量了捕食者与猎物的营养级差 值的变异性，因此杂食性越高，营养一致性越低。

杂食性和模块性是食物网结构复杂性的两个 重要方面。杂食性导致不同营养级之间的差别变得 模糊, 因而增加了食物网垂直结构的复杂性。模块 性不仅增大了食物网内种间相互作用的异质性, 还 增加了食物网水平和垂直结构的复杂性。如果没有 杂食性和模块性, 食物网很大程度上可简化为食物 链(即每个营养级由若干营养功能相似的物种组
成)。因此，阐明食物网中的杂食性和模块性特征， 是理解食物网结构复杂性的一个关键。

\section{2 食物网的水平和垂直多样性}

虽然物种多样性也可作为食物网的结构指标 之一, 但由于很多研究专门探讨食物网中多样性的 维持机制及其生态功能，因此本文将多样性作为一 类独立指标进行阐述。在食物网中, 多样性可由不 同指标、不同维度来刻画。指标上，可基于物种数 和连接度来计算。特别地, 生态系统中的所有物种 的总数可以表征总的物种多样性, 所有连接的总数 可以表征总的种间关系多样性。维度上，食物网一 般可由二维平面图表示, 垂直高度表示物种的营养 级，即处于同一高度的物种属于同一营养级，因而 可从水平和垂直两个维度刻画食物网的多样性(图 2a)。水平多样性(horizontal diversity)刻画了某一营 养级内的物种多样性, 垂直多样性(vertical diversity) 则刻画了跨营养级的结构复杂性。后者可通过营养 级的数目来度量, 比如食物链长度、复杂食物网的 平均营养级或最高营养级(Duffy et al, 2007; Wang \& Brose, 2018)。

此外，Duffy等(2007)提出也可基于连接数来定 义水平和垂直多样性, 即水平生态位幅度 (horizontal niche breadth)和垂直生态位幅度(vertical niche breadth)。具体地, 水平生态位幅度由捕食者 (a)

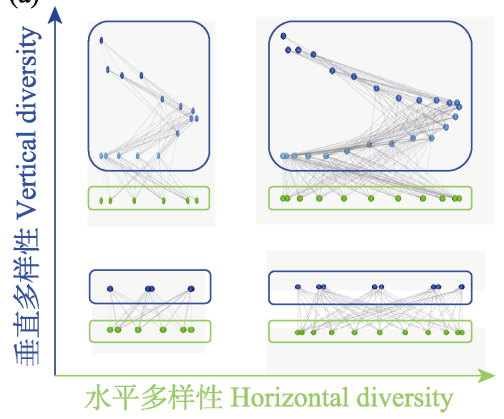

(b)

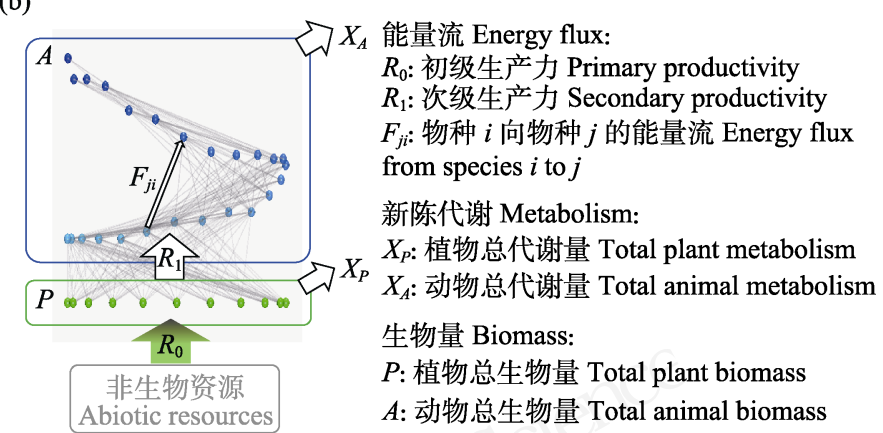

图2 食物网中的生物多样性(a)和功能(b)。在食物网中, 生物多样性可通过水平多样性(如同一营养级内的物种数)和垂直多 样性(如食物网的最高营养级)度量, 功能可通过营养级或物种之间的能量流、植物或动物的总代谢量和生物量刻画。其中, 非 生物资源与植物群落(绿色框)之间的能量流表征了初级生产力, 植物与动物群落(蓝色框)之间的能量流表征了次级生产力。 修改自Wang \& Brose (2018)和Schneider等(2016)。

Fig. 2 Food web diversity (a) and functioning (b). Biodiversity in food webs can be measured by both horizontal diversity (e.g. species richness within trophic levels) and vertical diversity (e.g. the maximum trophic level). Functioning can be measured by energy fluxes between two trophic levels or two species, total plant or animal metabolism, and total plant or animal biomass. Specifically, the energy flux from abiotic resource to plant communities (green box) represents the primary productivity, and that from plant communities to animal communities (blue box) represents the secondary productivity. Modified from Wang \& Brose (2018) and Schneider et al (2016). 
的广食性刻画, 即捕食者的总猎物种数; 垂直生态 位幅度由捕食者的杂食性刻画，即其猎物所分布的 营养级范围。但在本文中, 水平和垂直多样性特指基 于物种数的指标, 即分别指营养级内物种数和营养级 的数目, 而将广食性和杂食性作为网络结构指标。

\section{3 食物网的功能}

食物网的主要生态功能是能量传递和物质循 环。Elton (1927) 提出了数量金字塔 (numerical pyramid)的概念, 表征不同营养级间的种群大小分 布。这一概念后来被发展为能量金字塔(energy pyramid), 用来量化不同营养级之间的能量传递过 程(Lindeman, 1942; Trebilco et al, 2013)。其中 , 能量 金字塔的最底层为初级生产力, 表征了初级生产者 从非生物环境中固定的能量; 第二层为次级生产力, 量化了初级生产者向初级消费者传递的能量(图 2b)。近期研究对能量金字塔的概念做了扩展, 通过 计算任意两物种之间的能量流(energy flux)来刻画 食物网局部的功能特征, 从而对食物网能量动态做 出全面量化(Barnes et al, 2018) (图 2b)。具体地, 物 种之间的能量流可通过能量守恒法则计算得出, 即 物种的能量输入等于能量输出(de Ruiter et al, 1994)。由于需要对每一物种计算其能量平衡, 因此 该算法有较大的计算复杂度。最近 Gauzens 等(2019) 发展了一个矩阵算法, 并提供了 $\mathrm{R}$ 软件包 fluxweb, 只要给出食物网中每一物种的代谢速率和取食比 例, 则可推断整个网络的能量流。其中, 代谢速率 可由物种个体大小推算得到(Brown et al, 2004; Barnes et al, 2018)。除了能量流, 近期研究也常用植 物和动物的总生物量或总代谢量作为食物网的功 能指标(Schneider et al, 2016; Wang S et al, 2019; Maureaud et al, 2020)。

在物质循环方面, 解析碳、氮、磷等元素在生 态系统和大气之间的循环过程是生态系统研究的 核心内容(傅声雷等, 2019)。但近期食物网理论研究 大多只关注能量流动, 对物质循环过程的理论探讨 仍较少, 因此本文主要关注食物网结构和多样性对 能量过程的作用。整合食物网与物质循环将是未来 研究的一个重要方向(详见5.2节)。

\section{食物网结构如何影响物种多样性}

经典的竞争理论预测, 共存的物种数不会超过 限制资源的种类(Tilman, 1982)。但如果竞争同一资
源的两物种有一个共同的捕食者，且二者在资源利 用和抵御捕食上存在权衡关系，则可实现共存(Holt et al，1994)。这是由于两个竞争物种不仅在资源获 取上形成“利用性竞争” (exploitative competition), 还由于捕食者的存在而形成“似然竞争” (apparent competition), 后者为两个物种构建了新的生态位, 从而促进物种共存。Chesson和Kuang (2008)基于现 代物种共存理论进一步揭示, 物种在资源利用或抵 御捕食方面的差异性可通过类似机制促进共存，因 此利用性竞争和似然竞争在影响物种共存上具有 一定的对称性。

在前期基于竞争或捕食研究的基础上，近期的 理论研究探讨了复杂食物网内的物种多样性维持。 Brose (2008)利用复杂食物网研究了动物群落对植 物物种共存的影响, 发现即使只有一种非生物营养 资源，食物网也可以维持几十种植物的稳定共存 (也见Wang \& Brose, 2018)。其原因是, 动物群落形 成的复杂食物网改变了植物群落的生物环境, 极大 拓展了植物群落的生态位空间，由于不同植物与食 物网中的不同动物形成营养关系，这种非对称的 “营养生态位” (trophic niche)促进了植物种之间的 共存。这一理论预测得到了近期实验的支持。如Wang $\mathrm{L}$ 等(2019)在我国内蒙古草地开展的放牧实验发现， 混合放牧有利于维持更高的草地物种多样性。

对食物网整体而言，网络结构对多样性维持有 重要影响, 这里主要关注杂食性和模块性结构。

Stouffer和Bascompte (2010)研究了复杂食物网中四 种不同的三物种组件(比如食物链、竞争、似然竞争、 杂食性)对多样性维持的作用。结果表明，食物网中 的杂食性组件越多，物种多样性越高，因而杂食性 有利于复杂食物网中的多样性维持。Wang $S$ 等 (2019)考虑了一种特定的杂食性结构, 即集团内捕 食(intraguild predation), 通过模拟去除食物网中的 集团内捕食, 发现该结构有利于维持更高的物种多 样性。该研究结合五物种的简单网络和复杂食物网 揭示了其作用机制: 集团内捕食促使能量在营养级 内部停留，减缓了能量从低营养级到高营养级的传 递, 从而可降低高营养级物种的下行调控, 最终增 强了整个食物网的种群稳定性和多样性维持。此外, 模块性使得食物网中不同物种的相互作用局部化, 从而减弱了物种之间的交互作用强度，同时也减弱 了干扰在食物网中的传递。因此, 模块性结构最终 
更有利于复杂食物网中的物种多样性维持和稳定 性(Thébault \& Fontaine, 2010; Stouffer \& Bascompte, 2011)。这一理论结果为理解自然界食物网中相对较 高的模块性(相比于互惠网络)提供了一种可能思路 (Thébault \& Fontaine, 2010)。

食物网中的连接度也影响物种多样性的维持。 早期观点认为, 物种连接度越高, 其灭绝引起的次 级灭绝就越多, 因此食物网中连接度较高的物种是 多样性维持的关键(Dunne et al, 2002)。但该结论是 基于静态的食物网结构模型得出的, 并未考虑种群 动态以及物种间的交互作用与反馈(Box 1)。Zhao等 (2016)结合食物网结构和种群动态模型发现, 对物 种多样性维持起重要作用的是能量流更大的物种 (也即总能量输入或输出更多的物种)、而非连接度 更高的物种。此外, 物种性状也可通过改变种群动 态和种间关系来影响食物网的物种多样性维持。基 于个体大小的食物网模型表明, 捕食者一猎物的个 体大小比例对多样性维持具有重要作用(Brose et al, 2006)。具体地，捕食者相对于猎物的个体大小比例 越大, 则营养级间的能量流相对越缓慢, 从而可增 强种群稳定性和食物网的物种多样性。

\section{食物网结构与多样性如何影响生态系统功}

食物网的复杂结构为生态系统内的物质和能 量流动提供了多样化的渠道, 从而可能影响生态系 统功能。解析食物网结构和多样性对生态系统的调 控作用, 是理解生态系统运行机制的关键, 也是整 合群落生态学和生态系统生态学的重要途径 (Thompson et al, 2012)。本节概述了有关网络结构和 物种多样性如何影响食物网功能维持的最新进展。

\section{1 食物网结构与生态系统功能}

在生物、社会、经济等不同领域的网络中, 网 络结构都对维持相应系统的功能具有重要意义 (Strogatz, 2001), 食物网中亦然。早期关于营养级联 (trophic cascade)的研究, 为理解食物网结构如何影 响生态系统功能提供了重要认识。营养级联刻画了 食物链中的顶级捕食者对低营养级物种的间接作 用。特别地, 顶级捕食者通过下行级联效应可影响 初级生产者, 而后者又通过上行效应(bottom-up effect)对高营养级物种产生反馈。过去几十年关于 营养级联开展了大量研究, 其中涉及的几个重要问 题包括: 营养级联在水生和陆生生态系统中是否都
存在、其强度如何(Strong, 1992; Polis et al, 2000); 营养级联在生态系统内和不同生态系统之间的作 用机制是什么(Knight et al, 2005; Trussell et al, 2006; Leroux \& Loreau, 2008); 顶级捕食者灭绝可能产生 怎样的级联效应(Estes et al, 2011)等。孙书存(2017) 一文对营养级联理论作了深入阐述。

虽然营养级联效应在食物链模型中得到了很 好的支持, 但在复杂食物网中, 杂食性可能大大减 弱营养级联的作用。杂食性使得顶级捕食者与低营 养级物种间存在多种连接路径, 不同路径上的级联 作用可能相互抵消，从而减弱捕食者对低营养级的 调控(Polis \& Strong, 1996)。特别地，杂食性可改变 生物量的分布。食物链中顶级捕食者的跨营养级取 食，可导致生物量在营养级间的分布从金字塔型变 为“沙漏”型(Woodson et al, 2018)。复杂食物网中的 集团内捕食(一种杂食性结构)可减弱高营养级的下 行效应(top-down effect)作用，从而促进低营养级物 种的生物量积累，而后者又通过上行效应增加高营 养级的总生物量(Wang S et al, 2019)。然而，当外界 生产力或营养水平较低时, 集团内捕食不利于能量 或物质向高营养级传递，从而导致高营养级的总生 物量降低。杂食性表征了捕食者在垂直方向上的生 态位幅度, 而其在水平方向上的生态位幅度则由广 食性刻画。捕食者的广食性可增强猎物群落内的似 然竞争, 从而改变种群动态和生态系统功能。在单 营养级群落中，物种之间的利用性竞争可减弱生态 位互补作用(niche complementarity; Loreau，2010), 不利于生态系统功能的维持。Poisot等(2013)发现, 类似于利用性竞争，多营养级系统中的似然竞争对 生态系统功能有类似的负作用，提出了营养互补假 说(trophic complementarity hypothesis)。具体地, 给 定一个植物群落, 食草动物的广食性越高, 则植物 种之间的似然竞争越强, 最终植物群落的生物量和 生产力越低。

虽然关于食物网的研究有很长的历史, 但以往 研究较多关注食物网结构与稳定性的关系, 对网络 结构如何影响功能的认识仍较零散。特别地, 在众 多的食物网结构和功能指标(见本文第1节)中, 不同 结构指标与功能指标之间有何关联, 仍缺乏系统认 识(Thompson et al, 2012)。虽然如此，近期研究关于 食物网多样性对生态系统功能的作用取得了一系 列新进展，见3.2节。 


\section{2 食物网多样性与生态系统功能}

生物多样性与生态系统功能是近三十年来生 态学领域的研究热点, 为理解生物多样性丧失的生 态后果提供了重要认识。虽然最早的生物多样性实 验之一就研究了多营养级系统中的生物多样性对 生态系统过程的影响(Naeem et al, 1994), 但后期多 样性实验大多考虑单营养级系统, 尤其是植物群 落。这些实验表明, 植物多样性可促进生态系统初 级生产力, 作用机制可归纳为物种间的生态位互补 或竞争产生的选择效应(selection effect; Loreau, 2010; Tilman et al, 2014)。然而, 为了理解自然界中 的物种丧失的可能后果, 需要在食物网框架下研究 生物多样性与功能的关系, 这一方面是因为自然生 态系统中的物种都受到营养级间的相互作用, 另一 方面是因为高营养级物种面临更大的灭绝风险。虽 然早期食物网研究较少关注多样性与生态系统功 能的关系, 但过去20年在这一问题上取得了重要进 展, 以下从水平多样性和垂直多样性两方面阐述。

水平方向上, 近期研究主要从资源获取和抵抗 能力两方面探讨了营养级内的水平多样性的作用。 首先, 高营养级内的水平多样性可促进该营养级的 能量获取效率和生物量, 降低资源营养级的生物量 (Ives et al, 2004; Duffy et al, 2007)。然而, 相比于植 物多样性, 高营养级内的水平多样性对功能的促进 作用可能较弱, 这是因为不同于植物对非生物资源 的获取, 高营养级对低营养级的取食会产生较强的 下行调控, 从而引起种群波动, 甚至导致低营养级 物种的灭绝(Rosenzweig, 1971; Duffy et al, 2007)。 Cardinale等(2006)基于111项生物多样性实验的整 合分析却表明, 植物、食草动物、捕食者、分解者 等不同营养类群的水平多样性均具有正作用, 即促 进自身营养级的生产力和生物量并降低其资源营 养级的浓度或生物量; 与植物多样性相比, 其他三 个营养级的水平多样性具有相同强度的正作用。这 可能是由于该整合分析中的动物群落大多是下行 作用相对较弱的无脊椎动物类群(Borer et al, 2005), 也可能是由于营养级内多样性的增加引起了更多 的集团内捕食, 从而减弱了对低营养级的下行调控 (Wang S et al, 2019)。此外, 高营养级的存在可定性 或定量地改变营养级内水平多样性的作用(Thébault \& Loreau, 2003; Jiang \& Pu, 2009), 但以往的理论和 实验结果并未得出一致性结论, 而是发现高营养级
的作用受水平和垂直生态位幅度、物种在种群增长 和抵抗捕食上是否存在权衡关系等因素影响(Duffy et al, 2007)。另一方面, 营养级内的水平多样性可增 强该营养级对其捕食者群落的抵抗能力。比如, 多 样性更高的植物群落有更大的概率包含对食草动 物抵抗力强的物种, 从而降低食草动物的取食压力 (Hillebrand \& Cardinale, 2004)。对于寄主-寄生物系 统而言, 寄主的多样性可通过降低寄生物的传播速 率、抑制被感染的寄主种群等方式产生“稀释作用” (dilution effect), 降低寄主的被感染风险(Civitello et $\mathrm{al}, 2015)$ 。总而言之, 对某一特定营养级而言, 水平 多样性可促进其对低营养级的资源获取效率以及 对高营养级的抵抗力。这一作用可通过级联作用传 递到更高或更低的营养级, 但由于杂食性的存在和 中间营养级行为方式的调整, 多样性作用的强度可 能随营养级间的距离增加而大大减弱(Duffy et al, 2007; Haddad et al, 2009)。

垂直方向上, 食物网的最高营养级(即顶级捕 食者)可通过直接或间接的下行作用影响整个食物 网中的种群动态并最终影响初级生产者的生产力 (Wang \& Brose, 2018; Eisenhauer et al, 2019)。经典 的营养级联理论预测, 顶级捕食者抑制其猎物营养 级以及其他与其距离为奇数的营养级, 而释放与其 距离为偶数的营养级。因此, 随食物链长度增加, 初级生产者的生产力和生物量呈上升与下降交替 的波动变化(Loreau, 2010)。近期研究探讨了复杂食 物网模型中垂直多样性的作用, 得出了不同的预测 结果。Wang和Brose (2018)发现在复杂食物网中, 初 级生产力随最高营养级增加呈单调的指数增长, 提 出了“垂直多样性假说” (vertical diversity hypothesis)。食物链和复杂食物网模型之所以给出 了不一致的预测结果, 是因为食物链中存在较强的 营养级联作用, 而复杂食物网中的杂食性使得营养 级联的作用大大减弱(Polis \& Strong, 1996; Wang \& Brose, 2018)。在复杂食物网中, 垂直多样性的作用 机制可从两方面理解: 一方面, 复杂的营养结构可 为植物物种提供更多样的生态位, 促进了营养互补 效应(Poisot et al, 2013), 进而增强植物群落的营养 吸收效率。另一方面, 动物群落的捕食作用可改变 植物群落的性状组成, 从而通过选择效应影响植物 多样性与初级生产力的关系(Schneider et al, 2016)。 特别地, 更高的垂直多样性选择个体更大的植物种, 
而由于单位质量的新陈代谢速率随个体大小增加 而减小, 因此动物群落的选择作用可提高植物群落 的营养利用效率(Wang \& Brose, 2018)。

综上, 水平多样性有利于相应的营养级更有效 地从低营养级获取资源和抵抗高营养级的捕食, 垂 直多样性可通过下行调控作用促进初级生产力。类 似于单营养级系统中的多样性作用(Tilman et al, 2014), 食物网中的水平和垂直多样性的作用机制 也可通过互补效应和选择效应来认识, 但两种效应 的具体实现机制需从营养调控角度来理解。目前整 合水平和垂直多样性的研究仍较少, 但理论模型表 明, 食物网的总物种多样性可提高生态系统生产 力、生物量、总代谢速率等多方面的功能(Schneider et al, 2016; Maureaud et al, 2020)。

\section{全球变化如何影响食物网结构、多样性与}

全球环境变化和生物入侵等因素可能改变食 物网的结构和动态(Tylianakis et al, 2008; Sentis et al, 2014)。Tylianakis等(2008)基于对688篇文献的综述 分析表明, 气候和土地利用变化、富营养化 (eutrophication)、生物入侵等因素会显著改变不同 类型的种间作用关系, 包括植食、捕食、竞争、互 惠等, 但改变的方向在不同系统中存在较大差异。 深入理解全球变化对食物网的结构与功能的影响, 是预测生态系统对全球变化响应的基础。本节重点 介绍近期研究关注较多的两类全球变化因子, 即气 候变暖和富营养化。

\section{1 气候变暖}

气候变暖可通过多种方式影响食物网结构、动 态和功能。很多研究表明, 气候变化可引起物种在 空间分布范围和物候上的变化(Parmesan \& Yohe, 2003)。由于不同物种的响应往往存在差异, 可能引 起某些已有的种间关系的消失以及新的种间关系 的出现, 从而引起食物网结构的重建(Song et al, 2020)。不同子群落间的响应也可能存在差异, 比如 在陆地生态系统中, 增温可能引起地上食物网结构 发生较大变化, 而地下食物网结构相对稳健(Thakur, 2020)。认识气候变化下的“新兴种间关系” (novel interactions)和“新兴群落” (novel community) 是预测 生态系统结构变化的关键 (Gilman et al, 2010; Guiden et al, 2019)。

增温可引起食物网中物种性状、种群动态和种
间作用强度的变化，从而影响物种多样性维持。很 多研究表明, 增温可导致生物个体变小(Daufresne et al, 2009)。由于个体大小对种群过程和种间关系 有重要影响，因此增温可间接影响食物网动态和多 样性维持(Brown et al, 2004)。此外，增温还可直接 影响种群动态与种间关系。一方面, 生态学代谢理 论预测，增温可加速个体生长、新陈代谢和死亡等 过程(Brown et al, 2004); 另一方面，增温还可增大 捕食者的攻击速率、降低其对猎物的处理时间，从 而增加整体捕食速率(Box 1; Rall et al，2012; Fussmann et al, 2014)。虽然增温可同时增加捕食者 的捕食速率和代谢速率，但经验分析显示前者增加 的幅度往往小于后者，所以增温下捕食者的能量需 求更难满足，从而面临更大的灭绝风险(Fussmann et al, 2014)。因此，增温可能导致物种多样性丧失和 食物网营养级的下降(Zhang et al, 2017)。O'Gorman 等(2019)利用冰岛14个地热增温的溪流食物网数据, 证实增温可降低总物种数和垂直多样性。该研究还 发现，增温降低了食物网中的连接度，从而增大次 级灭绝风险。然而，增温并未导致该系统中个体大 小的下降，反而增大了消费者的平均个体大小，这 可能是由于在该系统中生产力也在随温度增加而 增加(O’Gorman et al, 2017)。因此，理解增温的作用 需考虑其与其他全球变化因子的交互作用。Binzer 等(2016)基于经验数据发展了一个参数化的食物网 模型，该模型预测，增温在生产力较高的地区可增 加物种多样性, 而在生产力较低的地区降低物种多 样性。

通过改变网络结构、多样性和种间作用强度, 增温最终可影响生态系统功能。多个实验研究表明, 增温可引起生态系统初级生产力的增加, 进而影响 不同营养级间的生物量分布(Petchey et al，1999; O’Connor et al, 2009)。基于海洋和溪流食物网的研 究均发现, 增温加强了消费者的下行调控作用, 促 使生物量在营养级间的分布向倒金字塔型转化 (O’Connor et al, 2009; O’Gorman et al, 2019)。然而, 增温可能同时改变生产者或消费者的物种组成，从 而影响营养级间的能量传递和下行调控。Petchey等 (1999)的微宇宙实验发现，增温引起高营养级物种 灭绝，因此减弱了下行调控作用，导致初级生产者 的生物量增加。Ullah等(2018)利用室内试验模拟了 增温和 $\mathrm{CO}_{2}$ 增加对海洋食物网的作用, 发现增温可 
引起初级生产者中的蓝细菌生物量增加。由于蓝细 菌可产生毒素和化感物质, 因而降低了生产者与食 草动物之间的营养传递效率(trophic efficiency), 最 终导致生产者生物量增加, 而食草动物和食肉动物 营养级的生物量下降。

\section{2 富营养化}

富营养化对多样性的作用可从两方面理解。一 方面, 富营养化可增加初级生产力, 并通过上行作 用传递到高营养级(Lindeman, 1942), 从而有利于 维持更高的物种数和垂直多样性(Post, 2002; Moore \& de Ruiter, 2012)。但另一方面, 富营养化引起的高 营养级的生物量增加可增强下行调控作用, 从而降 低整个系统的稳定性。特别地，过高的营养富集可 导致捕食者-猎物系统发生震荡, 从而增大物种灭 绝的风险，也即经典的“富集悖论”假说(paradox of enrichment; Rosenzweig, 1971)。基于这两方面作用, 理论模型预测：富营养化可增加营养贫㾑地区的物 种多样性，而降低营养富集地区的多样性(Rall et al, 2008; Rip \& McCann, 2011)。进一步地, 理论分析还 预测富营养化与增温之间存在交互作用: 增温可缓 解富营养化的负作用, 从而在高温环境下富营养化 促进物种多样性(Binzer et al, 2016)。

如上所述, 富营养化可增加初级生产力, 进而 促进高营养级的生产力和生物量。此外, 富营养化 可增加生产者中的营养元素含量(如氮和磷), 从而 提高生产者和初级消费者之间的营养传递效率 (Sterner \& Elser, 2002)。类似于增温, 富营养化也可 能引起物种组成发生变化, 比如增加抵抗力强的物 种的比例，从而限制能量向高营养级传递。Davis等 (2010)利用溪流食物网系统开展了为期5年的营养 添加实验, 结果发现, 在实验初期, 初级和次级消 费者的生物量和生产力都增加, 体现了溪流食物网 较高的能量传递效率; 但在实验后期, 虽然初级消 费者的生物量和生产力都在增加, 但次级消费者的 生产力却开始下降。这是由于, 初级消费者生物量 的增加主要来自个体较大的物种, 而次级消费者只 能取食个体较小的物种, 从而导致初级和次级消费 者之间的营养传递效率降低。

\section{研究展望}

食物网是生态学领域的一个经典研究议题。过 去几十年中, 关于食物网的理论和经验研究都取得
了重要进展, 特别是近期研究将早期的简单网络分 析拓展至复杂食物网。本文围绕食物网的结构、多 样性和功能, 对近期基于复杂食物网的理论进展进 行了概述。为了进一步深入理解食物网结构与功能 的关系及其各自的形成和维持机制，未来研究需进 一步加强食物网理论与功能性状、化学计量理论、 与其他生态网络的整合、并拓展其空间和时间尺度, 从而实现对生态系统复杂性的整体认识。

\section{1 物种功能性状}

物种功能性状对种群动态和食物网结构与功 能有重要影响。Gravel等(2016)提出在食物网研究中, 可根据物种性状的作用将其分成三类：(1)拓扑性状, 即影响网络结构或捕食关系存在与否的性状, 如昆 虫口器的形状; (2)消费性状, 即影响植食或捕食速 率的性状，如资源物种中营养组分或元素的含量; (3)生活史性状, 即影响种群增长或死亡的性状, 如 繁殖力和成熟时间。此分类只是基于性状作用的一 个划分，很多性状同时具有多方面作用，因此可被 归为多类。比如，目前食物网文献中研究最多的性 状是个体大小，它不仅可以预测种间捕食关系的有 无(Brose et al, 2019)、捕食速率(Rall et al, 2012)、种 群增长速率(Brown et al, 2004), 还有助于理解食物 网整体的结构复杂性(Laigle et al, 2018)和生态系统 功能(Schneider et al, 2016; Wang \& Brose, 2018)。因 此加强食物网功能性状研究, 有利于进一步整合食 物网结构、动态与功能。

\section{2 食物网与化学计量学}

目前的食物网理论大多基于种群动态模型，因 此可以较好地刻画物种在数量方面的特征, 如种群 大小和生物量。但很多研究表明, 捕食关系以及种 群动态都受到生物化学计量属性、营养元素含量等 质量特征的影响(Sterner \& Elser, 2002; Burian et al, 2020)。比如，食草动物取食营养含量高的植物时有 更高的同化效率(Sterner \& Elser, 2002), 对氮和钠 元素的需求可促进集团内捕食的发生(Denno \& Fagan, 2003; Clay et al, 2017)。在两物种或三物种的 食物链模型中考虑食物质量的影响, 可显著改变捕 食者-猎物动态、物种共存条件、能量传递效率等 (Elser et al, 2012; Peace, 2015)。如何在复杂食物网 中同时考虑数量与质量的调控作用, 是今后食物网 理论发展的一个重要方向。此类模型将促进生物地 球化学循环、化学计量、种群动态、食物网等研究 
领域的整合, 可为理解生态系统过程与功能提供新 的认识(Welti et al, 2017)。

\section{3 食物网与其他生态网络的整合}

自然群落中物种之间除了捕食关系, 还存在互 惠(mutualism)、寄生(parasitism)、竞争等种间作用。 目前越来越多的研究尝试用网络方法将不同的种 间作用整合起来(Fontaine et al，2011; Mougi \& Kondoh, 2012), 比如Pocock等(2012)构建了英格兰 西南部农场560种动植物之间的生态网络, 包含捕 食、寄生、传粉、种子传播等种间关系。理论分析 表明, 种间作用的多样性对生态系统稳定性的维持 有重要作用, 比如捕食和互惠关系的同时存在可促 进复杂生态系统的稳定性(Mougi \& Kondoh, 2012)。 另外, 当外界生物或非生物条件发生改变, 种间关 系也可能改变甚至相互转化(Holland \& DeAngelis, 2009)。一方 面, 环境条件的变化可能改变种间关系, 比如近期有实验发现, 随着环境营养浓度的增加, 两种酵母菌株可从低营养条件下的互惠关系转化 为高营养条件下的竞争或寄生关系(Hoek et al, 2016)。另一方面, 物种之间可能存在高阶作用, 即 某一物种的存在可定量或定性地改变其他物种之 间的关系(Goudard \& Loreau, 2008; Kéfi et al, 2012)。在生态网络研究中整合不同类型的种间关系 及其在生物和非生物条件下的改变与转化是一重 要新兴领域。

\section{4 集合食物网理论}

空间过程对生物多样性和生态系统功能的维 持具有重要作用。近20年来, 集合群落理论为理解 生境异质性、扩散等因素的作用提供了新的研究框 架, 大大增进了对生境破坏的生态效应的认识 (Leibold \& Chase, 2018)。然而以往集合群落模型大 多关注单营养级的竞争群落, 且通常假定物种在不 同斑块之间随机扩散。未来研究亟待加强对集合食 物网模型的研究, 其中需特别考虑扩散方式和空间 异质性(Leibold \& Chase, 2018)。与竞争群落不同, 食物网中的不同营养级由于个体大小等性状上的 差异, 往往具有不同的扩散速率(McCann et al, 2005; Hirt et al, 2017), 因此模型中需考虑扩散在营 养级之间的差异。此外, 对食物网中的物种来说, 其扩散可能受其猎物和捕食者的影响，即种群倾向 于向猎物密度更高、捕食者密度更低的斑块扩散 (Fronhofer et al, 2018)。在集合食物网模型中考虑更
为现实的扩散情景, 有利于预测气候变化下食物网 结构和多样性的响应 (Thompson \& Gonzalez, 2017)。

\section{5 进化模型}

虽然食物网模型可以有效地刻画自然界食物 网的结构指标, 但这些模型无法解释这些结构是如 何产生的。进化模型则可通过模拟物种属性(比如个 体大小、捕食宽度等)的演化过程, 帮助我们理解复 杂的食物网结构如何从物种进化过程中涌现出来 (Loeuille \& Loreau, 2005; Ingram et al, 2009)。在全 球变化背景下, 生态和进化过程的反馈对于食物网 结构和功能有重要作用。比如, 某一物种的适应性 进化不仅对其自身的种群维持起作用(比如进化拯 救效应; evolutionary rescue), 还对食物网中与其直 接相关的捕食者和猎物以及间接相关的其他物种 产生影响(Loeuille, 2019)。因此，构建食物网的生态 -进化模型有利于理解全球变化下生态系统的适应 性响应。

\section{参考文献}

Allesina S, Alonso D, Pascual M (2008) A general model for food web structure. Science, 320, 658-661.

Barnes AD, Jochum M, Lefcheck JS, Eisenhauer N, Scherber C, O’Connor MI, de Ruiter P, Brose U (2018) Energy flux: The link between multitrophic biodiversity and ecosystem functioning. Trends in Ecology \& Evolution, 33, 186-197.

Bascompte J (2009) Disentangling the web of life. Science, 325, 416-419.

Binzer A, Guill C, Rall BC, Brose U (2016) Interactive effects of warming, eutrophication and size structure: Impacts on biodiversity and food-web structure. Global Change Biology, 22, 220-227.

Borer ET, Seabloom EW, Shurin JB, Anderson KE, Blanchette CA, Broitman B, Cooper SD, Halpern BS (2005) What determines the strength of a trophic cascade? Ecology, 86, 528-537.

Brose U, Williams RJ, Martinez ND (2006) Allometric scaling enhances stability in complex food webs. Ecology Letters, 9, 1228-1236.

Brose U (2008) Complex food webs prevent competitive exclusion among producer species. Proceedings of the Royal Society B: Biological Sciences, 275, 2507-2514.

Brose U, Archambault P, Barnes AD, Bersier L-F, Boy T, Canning-Clode J, Conti E, Dias M, Digel C, Dissanayake A, Flores AVA, Fussmann K, Gauzens B, Gray C, Häussler J, Hirt MR, Jacob U, Jochum M, Kéfi S, McLaughlin O, MacPherson MM, Latz E, Layer-Dobra K, Legagneux P, Li YH, Madeira C, Martinez ND, Mendonça V, Mulder C, 
Navarrete SA, O’Gorman EJ, Ott D, Paula J, Perkins D, Piechnik D, Pokrovsky I, Raffaelli D, Rall BC, Rosenbaum B, Ryser R, Silva A, Sohlström EH, Sokolova N, Thompson MSA, Thompson RM, Vermandele F, Vinagre C, Wang S, Wefer JM, Williams RJ, Wieters E, Woodward G, Iles AC (2019) Predator traits determine food-web architecture across ecosystems. Nature Ecology \& Evolution, 3, 919-927.

Brown JH, Gillooly JF, Allen AP, Savage VM, West GB (2004) Toward a metabolic theory of ecology. Ecology, 85, 1771-1789.

Burian A, Nielsen JM, Winder M (2020) Food quantity-quality interactions and their impact on consumer behavior and trophic transfer. Ecological Monographs, 90, e01395.

Cardinale BJ, Srivastava DS, Duffy JE, Wright JP, Downing AL, Sankaran M, Jouseau C (2006) Effects of biodiversity on the functioning of trophic groups and ecosystems. Nature, 443, 989-992.

Cattin MF, Bersier LF, Banašek-Richter C, Baltensperger R, Gabriel JP (2004) Phylogenetic constraints and adaptation explain food-web structure. Nature, 427, 835-839.

Chen YF, Tang Z, Li H, Han XM, Li YF, Hu C (2014) Research progress on ecosystem complexity-stability relationships based on soil food web. Acta Ecologica Sinica, 34, 2173-2186. (in Chinese with English abstract) [陈云峰, 唐政, 李慧, 韩雪梅, 李钰飞, 胡诚 (2014) 基于土壤食 物网的生态系统复杂性-稳定性关系研究进展. 生态学 报, 34, 2173-2186.]

Chesson P, Kuang JJ (2008) The interaction between predation and competition. Nature, 456, 235-238.

Civitello DJ, Cohen J, Fatima H, Halstead NT, Liriano J, McMahon TA, Ortega CN, Sauer EL, Sehgal T, Young S, Rohr JR (2015) Biodiversity inhibits parasites: Broad evidence for the dilution effect. Proceedings of the National Academy of Sciences, USA, 112, 8667-8671.

Clay NA, Lehrter RJ, Kaspari M (2017) Towards a geography of omnivory: Omnivores increase carnivory when sodium is limiting. Journal of Animal Ecology, 86, 1523-1531.

Cohen JE, Newman CM (1985) A stochastic theory of community food webs. I. Models and aggregated data. Proceedings of the Royal Society B: Biological Sciences, 224, 421-448.

Daufresne M, Lengfellner K, Sommer U (2009) Global warming benefits the small in aquatic ecosystems. Proceedings of the National Academy of Sciences, USA, 106, 12788-12793.

Davis JM, Rosemond AD, Eggert SL, Cross WF, Wallace JB (2010) Long-term nutrient enrichment decouples predator and prey production. Proceedings of the National Academy of Sciences, USA, 107, 121-126.

de Ruiter PC, Neutel AM, Moore JC (1994) Modelling food webs and nutrient cycling in agro-ecosystems. Trends in Ecology \& Evolution, 9, 378-383.
Denno RF, Fagan WF (2003) Might nitrogen limitation promote omnivory among carnivorous arthropods? Ecology, 91, 3114-3117.

Duffy JE, Cardinale BJ, France KE, McIntyre PB, Thébault E, Loreau M (2007) The functional role of biodiversity in ecosystems: Incorporating trophic complexity. Ecology Letters, 10, 522-538.

Dunne JA, Williams RJ, Martinez ND (2002) Network structure and biodiversity loss in food webs: Robustness increases with connectance. Ecology Letters, 5, 558-567.

Eisenhauer N, Schielzeth H, Barnes AD, Barry KE, Bonn A, Brose U, Bruelheide H, Buchmann N, Buscot F, Ebeling A, Ferlian O, Freschet GT, Giling DP, Hättenschwiler S, Hillebrand H, Hines J, Isbell F, Koller-France E, König-Ries B, de Kroon H, Meyer ST, Milcu A, Müller J, Nock CA, Petermann JS, Roscher C, Scherber C, Scherer-Lorenzen M, Schmid B, Schnitzer SA, Schuldt A, Tscharntke T, Türke M, van Dam NM, van der Plas F, Vogel A, Wagg C, Wardle DA, Weigelt A, Weisser WW, Wirth C, Jochum M (2019) A multitrophic perspective on biodiversity-ecosystem functioning research. Advances in Ecological Research, 61, $1-54$.

Elser JJ, Loladze I, Peace AL, Kuang Y (2012) Lotka re-loaded: Modeling trophic interactions under stoichiometric constraints. Ecological Modelling, 245, 3-11.

Elton CS (1927) Animal Ecology. Sidgwick and Jackson, London.

Estes JA, Terborgh J, Brashares JS, Power ME, Berger J, Bond WJ, Carpenter SR, Essington TE, Holt RD, Jackson JBC, Marquis RJ, Oksanen L, Oksanen T, Paine RT, Pikitch EK, Ripple WJ, Sandin SA, Scheffer M, Schoener TW, Shurin JB, Sinclair ARE, Soulé ME, Virtanen R, Wardle DA (2011) Trophic downgrading of planet Earth. Science, 333, 301-306.

Fontaine C, Guimarães PR Jr, Kéfi S, Loeuille N, Memmott J, van der Putten WH, van Veen FJF, Thébault E (2011) The ecological and evolutionary implications of merging different types of networks. Ecology Letters, 14, 1170-1181.

Fronhofer EA, Legrand D, Altermatt F, Ansart A, Blanchet S, Bonte D, Chaine A, Dahirel M, De Laender F, De Raedt J, di Gesu L, Jacob S, Kaltz O, Laurent E, Little CJ, Madec L, Manzi F, Masier S, Pellerin F, Pennekamp F, Schtickzelle N, Therry L, Vong A, Winandy L, Cote J (2018) Bottom-up and top-down control of dispersal across major organismal groups. Nature Ecology \& Evolution, 2, 1859-1863.

Fu SL, Zhang WX, Shao YH, Shi LL, Liu ZF (2019) Soil Ecology_Soil Food Web and Its Ecosystem Functioning. Science Press, Beijing. (in Chinese) [傅声雷, 张卫信, 邵 元虎, 时雷雷, 刘占锋 (2019) 土壤生态学一土壤食物 网及其生态功能. 科学出版社, 北京.]

Fussmann KE, Schwarzmüller F, Brose U, Jousset A, Rall BC (2014) Ecological stability in response to warming. Nature 
Climate Change, 4, 206-210.

Gauzens B, Legendre S, Lazzaro X, Lacroix G (2013) Food-web aggregation, methodological and functional issues. Oikos, 122, 1606-1615.

Gauzens B, Barnes A, Giling DR, Hines J, Jochum M, Lefcheck JS, Rosenbaum B, Wang S, Brose U (2019) Fluxweb: An R package to easily estimate energy fluxes in food webs. Methods in Ecology \& Evolution, 10, 270-279.

Gilman SE, Urban MC, Tewksbury J, Gilchrist GW, Holt RD (2010) A framework for community interactions under climate change. Trends in Ecology \& Evolution, 25, 325-331.

Goudard A, Loreau M (2008) Nontrophic interactions, biodiversity, and ecosystem functioning: An interaction web model. The American Naturalist, 171, 91-106.

Gravel D, Albouy C, Thuiller W (2016) The meaning of functional trait composition of food webs for ecosystem functioning. Philosophical Transactions of the Royal Society B: Biological Sciences, 371, 20150268.

Guiden PW, Bartel SL, Byer NW, Shipley AA, Orrock JL (2019) Predator-prey interactions in the anthropocene: Reconciling multiple aspects of novelty. Trends in Ecology \& Evolution, 34, 616-627.

Haddad NM, Crutsinger GM, Gross K, Haarstad J, Knops JMH, Tilman D (2009) Plant species loss decreases arthropod diversity and shifts trophic structure. Ecology Letters, 12, 1029-1039.

Hillebrand H, Cardinale BJ (2004) Consumer effects decline with prey diversity. Ecology Letters, 7, 192-201.

Hirt MR, Jetz W, Rall BC, Brose U (2017) A general scaling law reveals why the largest animals are not the fastest. Nature Ecology \& Evolution, 1, 1116-1122.

Hoek TA, Axelrod K, Biancalani T, Yurtsev EA, Liu JH, Gore J (2016) Resource availability modulates the cooperative and competitive nature of a microbial cross-feeding mutualism. PLoS Biology, 14, e1002540.

Holland JN, DeAngelis DL (2009) Consumer-resource theory predicts dynamic transitions between outcomes of interspecific interactions. Ecology Letters, 12, 1357-1366.

Holling CS (1959) Some characteristics of simple types of predation and parasitism. The Canadian Entomologist, 91, 385-398.

Holt RD, Grover J, Tilman GD (1994) Simple rules for interspecific dominance in systems with exploitative and apparent competition. The American Naturalist, 144, 741-771.

Ingram T, Harmon LJ, Shurin JB (2009) Niche evolution, trophic structure, and species turnover in model food webs. The American Naturalist, 174, 56-67.

Ives AR, Cardinale BJ, Snyder WE (2004) A synthesis of subdisciplines: Predator-prey interactions, and biodiversity and ecosystem functioning. Ecology Letters, 8, 102-116.

Jiang L, Pu Z (2009) Different effects of species diversity on temporal stability in single-trophic and multitrophic communities. The American Naturalist, 174, 651-659.

Johnson S, Domínguez-García V, Donetti L, Munoz MA (2014) Trophic coherence determines food-web stability. Proceedings of the National Academy of Sciences, USA, 111, 17923-17928.

Kalinkat G, Schneider FD, Digel C, Guill C, Rall BC, Brose U (2013) Body masses, functional responses and predator-prey stability. Ecology Letters, 16, 1126-1134.

Kéfi S, Berlow EL, Wieters EA, Navarrete SA, Petchey OL, Wood SA, Boit A, Joppa LN, Lafferty KD, Williams RJ, Martinez ND, Menge BA, Blanchette CA, Iles AC, Brose U (2012) More than a meal... integrating non-feeding interactions into food webs. Ecology Letters, 15, 291-300.

Knight TM, McCoy MW, Chase JM, McCoy KA, Holt RD (2005) Trophic cascades across ecosystems. Nature, 437, 880-883.

Krause AE, Frank KA, Mason DM, Ulanowicz RE, Taylor WW (2003) Compartments revealed in food-web structure. Nature, 426, 282-285.

Laigle I, Aubin I, Digel C, Brose U, Boulangeat I, Gravel D (2018) Species traits as drivers of food web structure. Oikos, 127, 316-326.

Leibold MA, Chase JM (2018) Metacommunity Ecology. Princeton University Press, Princeton NJ.

Leroux SJ, Loreau M (2008) Subsidy hypothesis and strength of trophic cascades across ecosystems. Ecology Letters, 11, 1147-1156.

Lindeman RL (1942) The trophic-dynamic aspect of ecology. Ecology, 23, 399-417.

Loeuille N, Loreau M (2005) Evolutionary emergence of size-structured food webs. Proceedings of the National Academy of Sciences, USA, 102, 5761-5766.

Loeuille N (2019) Eco-evolutionary dynamics in a disturbed world: Implications for the maintenance of ecological networks. F1000Research, 8, 97.

Loreau M (2010) From Populations to Ecosystems: Theoretical Foundations for a New Ecological Synthesis. Princeton University Press, Princeton NJ.

Maureaud A, Andersen KH, Zhang L, Lindegren M (2020) Trait-based food web model reveals the underlying mechanisms of biodiversity-ecosystem functioning relationships. Journal of Animal Ecology, 89, 1497-1510.

May RM (1973) Stability and Complexity in Model Ecosystems. Princeton University Press, Princeton NJ.

McCann KS, Rasmussen JB, Umbanhowar J (2005) The dynamics of spatially coupled food webs. Ecology Letters, 8, 513-523.

McCann KS (2012) Food Webs. Princeton University Press, Princeton NJ.

Moore JC, de Ruiter PC (2012) Energetic Food Webs: An Analysis of Real and Model Ecosystems. Oxford University Press, New York. 
Mougi A, Kondoh M (2012) Diversity of interaction types and ecological community stability. Science, 337, 349-351.

Naeem S, Thompson LJ, Lawler SP, Lawton JH, Woodfin RM (1994) Declining biodiversity can alter the performance of ecosystems. Nature, 368, 734-737.

O’Connor MI, Piehler MF, Leech DM, Anton A, Bruno JF (2009) Warming and resource availability shift food web structure and metabolism. PLoS Biology, 7, e1000178.

O’Gorman EJ, Zhao L, Pichler DE, Adams G, Friberg N, Rall BC, Seeney A, Zhang HY, Reuman DC, Woodward G (2017) Unexpected changes in community size structure in a natural warming experiment. Nature Climate Change, 7 , 659-663.

O’Gorman EJ, Petchey OL, Faulkner KJ, Gallo B, Gordon TA, Neto-Cerejeira J, Ólafsson JS, Pichler DE, Thompson MS, Woodward G (2019) A simple model predicts how warming simplifies wild food webs. Nature Climate Change, 9, 611-616.

Parmesan C, Yohe G (2003) A globally coherent fingerprint of climate change impacts across natural systems. Nature, 421, 37-42.

Peace A (2015) Effects of light, nutrients, and food chain length on trophic efficiencies in simple stoichiometric aquatic food chain models. Ecological Modelling, 312, 125-135.

Persson L, Leonardsson K, de Roos AM, Gyllenberg M, Christensen B (1998) Ontogenetic scaling of foraging rates and the dynamics of a size-structured consumer-resource model. Theoretical Population Biology, 54, 270-293.

Petchey OL, McPhearson PT, Casey TM, Morin PJ (1999) Environmental warming alters food-web structure and ecosystem function. Nature, 402, 69-72.

Pimm SL (1982) Food Webs. Springer, Dordrecht.

Pocock MJO, Evans DM, Memmott J (2012) The robustness and restoration of a network of ecological networks. Science, 335, 973-977.

Poisot T, Mouquet N, Gravel D (2013) Trophic complementarity drives the biodiversity-ecosystem functioning relationship in food webs. Ecology Letters, 16, 853-861.

Polis GA, Strong DR (1996) Food web complexity and community dynamics. The American Naturalist, 147, 813-846.

Polis GA, Sears ALW, Huxel GR, Strong DR, Maron J (2000) When is a trophic cascade a trophic cascade? Trends in Ecology \& Evolution, 15, 473-475.

Post DM (2002) The long and short of food-chain length. Trends in Ecology \& Evolution, 17, 269-277.

Rall BC, Guill C, Brose U (2008) Food-web connectance and predator interference dampen the paradox of enrichment. Oikos, 117, 202-213.

Rall BC, Brose U, Hartvig M, Kalinkat G, Schwarzmüller F, Vucic-Pestic O, Petchey OL (2012) Universal temperature and body-mass scaling of feeding rates. Philosophical Transactions of the Royal Society B: Biological Sciences, 367, 2923-2934.

Rip JMK, McCann KS (2011) Cross-ecosystem differences in stability and the principle of energy flux. Ecology Letters, 14, 733-740.

Rosenzweig ML (1971) Paradox of enrichment: Destabilization of exploitation ecosystems in ecological times. Science, 171, 385-387.

Schneider FD, Brose U, Rall BC, Guill C (2016) Animal diversity and ecosystem functioning in dynamic food webs. Nature Communications, 7, 12718.

Seibold S, Cadotte MW, MacIvor JS, Thorn S, Müller J (2018) The necessity of multitrophic approaches in community ecology. Trends in Ecology \& Evolution, 33, 754-764.

Sentis A, Hemptinne JL, Brodeur J (2014) Towards a mechanistic understanding of temperature and enrichment effects on species interaction strength, omnivory and food-web structure. Ecology Letters, 17, 785-793.

Song C, Ahn S, Rohr RP, Saavedra S (2020) Towards a probabilistic understanding about the context-dependency of species interactions. Trends in Ecology \& Evolution, 35, 384-396.

Sterner RW, Elser JJ (2002) Ecological Stoichiometry: The Biology of Elements from Molecules to the Biosphere. Princeton University Press, Princeton NJ.

Stouffer DB, Bascompte J (2010) Understanding food-web persistence from local to global scales. Ecology Letters, 13, 154-161.

Stouffer DB, Bascompte J (2011) Compartmentalization increases food-web persistence. Proceedings of the National Academy of Sciences, USA, 108, 3648-3652.

Strogatz SH (2001) Exploring complex networks. Nature, 410, 268-276.

Strong DR (1992) Are trophic cascades all wet? Differentiation and donor-control in speciose ecosystems. Ecology, 73, 747-754.

Sun SC (2017) Food-web structure and ecosystem functioning. In: Current Knowledge and Future Challenges (eds Yu ZL, Ge JP, Yu GR, Zhang DY, Fang JY, Kang L, Fu BJ, Jiao NZ), pp. 254-271. Higher Education Press, Beijing. (in Chinese) [孙书存 (2017) 食物网结构与生态功能. 见: 生 态学的现状与发展趋势 (于振良, 葛剑平, 于贵瑞, 张大 勇, 方精云, 康乐, 傅伯杰, 焦念志编), 254-271页. 高等 教育出版社, 北京.]

Thakur MP (2020) Climate warming and trophic mismatches in terrestrial ecosystems: The green-brown imbalance hypothesis. Biology Letters, 16, 20190770.

Thébault E, Loreau M (2003) Food-web constraints on biodiversity-ecosystem functioning relationships. Proceedings of the National Academy of Sciences, USA, 100, 14949-14954.

Thébault E, Fontaine C (2010) Stability of ecological 
communities and the architecture of mutualistic and trophic networks. Science, 329, 853-856.

Thompson RM, Hemberg M, Starzomski BM, Shurin JB (2007) Trophic levels and trophic tangles: The prevalence of omnivory in real food webs. Ecology, 88, 612-617.

Thompson RM, Brose U, Dunne JA, Hall RO, Hladyz S, Kitching RL, Martinez ND, Rantala H, Romanuk TN, Stouffer DB, Tylianakis JM (2012) Food webs: Reconciling the structure and function of biodiversity. Trends in Ecology \& Evolution, 27, 689-697.

Thompson PL, Gonzalez A (2017) Dispersal governs the reorganization of ecological networks under environmental change. Nature Ecology \& Evolution, 1, 0162.

Tilman D (1982) Resource Competition and Community Structure. Princeton University Press, Princeton NJ.

Tilman D, Isbell F, Cowles JM (2014) Biodiversity and ecosystem functioning. Annual Review of Ecology, Evolution, and Systematics, 45, 471-493.

Trebilco R, Baum JK, Salomon AK, Dulvy NK (2013) Ecosystem ecology: Size-based constraints on the pyramids of life. Trends in Ecology \& Evolution, 28, 423-431.

Trussell GC, Ewanchuk PJ, Matassa CM (2006) Habitat effects on the relative importance of trait- and density-mediated indirect interactions. Ecology Letters, 9, 1245-1252.

Turchin P (2003) Complex Population Dynamics: A Theoretical/Empirical Synthesis. Princeton University Press, Princeton NJ.

Tylianakis JM, Didham RK, Bascompte J, Wardle DA (2008) Global change and species interactions in terrestrial ecosystems. Ecology Letters, 11, 1351-1363.

Ullah H, Nagelkerken I, Goldenberg SU, Fordham DA (2018) Climate change could drive marine food web collapse through altered trophic flows and cyanobacterial proliferation. PLoS Biology, 16, e2003446.

Wang L, Delgado-Baquerizo M, Wang D, Isbell F, Liu J, Feng C, Liu J, Zhong Z, Zhu H, Yuan X, Chang Q, Liu C (2019) Diversifying livestock promotes multidiversity and multifunctionality in managed grasslands. Proceedings of the National Academy of Sciences, USA, 116, 6187-6192.

Wang S, Brose U (2018) Biodiversity and ecosystem functioning in food webs: The vertical diversity hypothesis. Ecology Letters, 21, 9-20.

Wang S, Brose U, Gravel D (2019) Intraguild predation enhances biodiversity and functioning in complex food webs. Ecology, 100, e02616.

Welti N, Striebel M, Ulseth AJ, Cross WF, DeVilbiss S, Glibert PM, Guo L, Hirst AG, Hood J, Kominoski JS, MacNeill KL, Mehring AS, Welter JR, Hillebrand H (2017) Bridging food webs, ecosystem metabolism, and biogeochemistry using ecological stoichiometry theory. Frontiers in Microbiology, 8, 1298 .

Williams RJ, Martinez ND (2000) Simple rules yield complex food webs. Nature, 404, 180-183.

Williams RJ, Martinez ND (2004) Limits to trophic levels and omnivory in complex food webs: Theory and data. The American Naturalist, 163, 458-468.

Woodson CB, Schramski JR, Joye SB (2018) A unifying theory for top-heavy ecosystem structure in the ocean. Nature Communications, 9, 23.

Xu GH, Li XY, Shi CH (2019) The complexity-stability relationship: Progress in mathematical models. Biodiversity Science, 27, 1364-1378. (in Chinese with English abstract) [徐光华, 李小玉, 施春华 (2019) 复杂性-稳定性研究: 数学模型的进展. 生物多样性, 27, 1364-1378.]

Yan C, Zhang Z (2014) Specific non-monotonous interactions increase persistence of ecological networks. Proceedings of the Royal Society B: Biological Sciences, 281, 20132797.

Yodzis P (1982) The compartmentation of real and assembled ecosystems. The American Naturalist, 120, 551-570.

Yodzis P, Innes S (1992) Body size and consumer-resource dynamics. The American Naturalist, 139, 1151-1175.

Zhang L, Takahashi D, Hartvig M, Andersen KH (2017) Food-web dynamics under climate change. Proceedings of the Royal Society B: Biological Sciences, 284, 20171772.

Zhao L, Zhang H, O’Gorman EJ, Tian W, Ma A, Moore JC, Borrett SR, Woodward G (2016) Weighting and indirect effects identify keystone species in food webs. Ecology Letters, 19, 1032-1040.

(责任编委: 张全国 责任编辑: 问文杰) 